\title{
$\alpha-\mathrm{MnO}_{2}$ 纳米棒/多孔碳正极材料的制备及水系锌离子电池性能研究
}

\author{
李燕丽于丹丹林森孙东飞* 雷自强 \\ (西北师范大学化学化工学院 生态功能高分子材料教育部重点实验室 兰州 730070)
}

\begin{abstract}
摘要 针对水系锌离子电池锰基正极材料存在比容量低、循环稳定性差等问题, 本工作利用水热法制备出棒状结构的 $\alpha-\mathrm{MnO}_{2}$, 通过柠檬酸钠高温碳化制备多孔碳, 进而通过超声分散等处理制备出 $\alpha-\mathrm{MnO}_{2} / \mathrm{PCSs}$ 复合材料. 三维的多孔网 络有助于提高电子导电性, 提供一个稳定的支撑; $\alpha-\mathrm{MnO}_{2}$ 纳米棒均匀地附着在多孔碳纳米片层表面, 有效地避免 $\alpha-\mathrm{MnO}_{2}$ 的团聚，从而提高锌离子传输效率. 得益于 $\alpha-\mathrm{MnO}_{2} / \mathrm{PCSs}$ 独特的结构优势, 将其作为锌离子电池正极材料, 在 电流密度为 $0.1 \mathrm{~A} \cdot \mathrm{g}^{-1}$ 的条件下循环 100 次后, 其可逆容量为 $350 \mathrm{mAh} \cdot \mathrm{g}^{-1}$, 在 $1 \mathrm{~A} \cdot \mathrm{g}^{-1}$ 的大的电流密度下, 经过 1000 圈 循环后, 容量可达 $160 \mathrm{mAh} \cdot \mathrm{g}^{-1}$, 展现了优异的循环稳定性能, 有望成为高性能锌离子电池的潜在正极材料.
\end{abstract}

关键词 水系锌离子电池; 正极材料; $\alpha-\mathrm{MnO}_{2}$ 纳米棒; 多孔碳纳米片; 电化学性能

\section{Preparation of $\alpha-\mathrm{MnO}_{2}$ Nanorods/Porous Carbon Cathode for Aqueous Zinc-ion Batteries}

\author{
Yanli Li Dandan Yu Sen Lin Dongfei Sun* Ziqiang Lei \\ (Key Laboratory of Eco-functional Polymer Materials of the Ministry of Education, College of Chemistry and \\ Chemical Engineering, Northwest Normal University, Lanzhou 730070, China)
}

\begin{abstract}
Aqueous zinc-ion batteries (ZIBs) have attracted more attention as large-scale energy storage technology due to their high safety, low cost and environmental benignity. To date, numerous cathodes based on manganese dioxide, vanadium dioxide, and polyanionic compounds have been reported. Among them, Manganese oxides have the advantages of low cost, non-toxicity, abundant materials and high working voltage, have been widely explored as promising cathodes for zinc ion batteries. Among them, $\mathrm{MnO}_{2}$ cathodes are particularly desirable candidates for commercialization owing to their tunnel structure and affordability. $\alpha-\mathrm{MnO}_{2}$ has $(1 \times 1)$ and $(2 \times 2)$ tunnel structures, and $\mathrm{Zn}^{2+}$ can rapidly inserted and deserted in the tunnel. However, the capacity of manganese dioxide is fast decaying with the cycles due to the poor conductivity of $\mathrm{MnO}_{2}$, which limits its electrochemical performance. Herein, $\alpha-\mathrm{MnO}_{2}$ nanorods are uniformly distributed on the surface of porous carbon nanosheets network (PCSs) by a simple hydrothermal/dispersion method strategy. In the $\alpha-\mathrm{MnO}_{2} / \mathrm{PCSs}$ architecture, the $\alpha-\mathrm{MnO}_{2}$ nanorods and $\alpha-\mathrm{MnO}_{2} / \mathrm{PCSs}$ composite were characterized by X-ray diffraction (XRD), scanning electron microscopy (SEM), high-resolution transmission electron microscopy (HRTEM), etc. The results testified that $\alpha-\mathrm{MnO}_{2} / \mathrm{PCSs}$ nanorods were firmly adhered on the surface of porous carbon nanosheets, which can effectively avoid the stacking of $\alpha-\mathrm{MnO}_{2}$ nanorods, while the high conductive carbon network can improve the electrical conductivity of the composite. The porous network can provide effective electron transfer channels, provide stable hosts for fast $\mathrm{Zn}^{2+}$ extraction/insertion, and prevent the $\alpha-\mathrm{MnO}_{2}$ nanorods from stacking each other. Benefiting from the unique porous structure and high conductive network, the $\alpha-\mathrm{MnO}_{2} / \mathrm{PCSs}$ hybrid shows high reversibility capacity, good rate performance, and outstanding cycling stability. Specifically, $\alpha-\mathrm{MnO}_{2} / \mathrm{PCSs}$ exhibits high reversible capacity of $350 \mathrm{mAh} \cdot \mathrm{g}^{-1}$ after 100 cycles at $0.1 \mathrm{~A}^{\circ} \mathrm{g}^{-1}$ and maintains

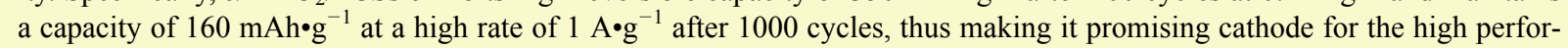
mance ZIBs.
\end{abstract}

Keywords aqueous zinc ion battery; cathode material; $\alpha-\mathrm{MnO}_{2}$ nanorods; porous carbon nanosheets; electrochemical performance

\section{1 引言}

随着便携式移动电子设备和电动汽车设备的快速 发展, 对储能装置提出了更高的要求. 在众多储能设备 中, 锂离子电池由于具有高的能量密度和大的电位窗口
而被广泛研究和应用 ${ }^{[1-4]}$. 然而, 由于锂资源的储量有 限、价格高和安全性低等问题的日益突出，探索具有高 容量、低成本和高安全性的先进的储能技术迫在眉睫 ${ }^{[5]}$. 可充电水系电池具有安全性好、成本低和离子电导率高 等优点. 尤其是锌离子电池(ZIBs)具有理论容量高( 820

\footnotetext{
* E-mail: dongfeisun@nwnu.edu.cn

Received September 16, 2020; published December 17, 2020.
}

Supporting information for this article is available free of charge via the Internet at http://sioc-journal.cn

Project supported by the National Natural Science Foundation of China (51863019), the Natural Science Foundation of Gansu Province of China (20JR10RA108) and the Innovation Fund of Gansu Universities (2020A-013).

项目受国家自然科学基金(51863019)、甘肃省自然科学基金(20JR10RA108)和甘肃省高等学校创新基金项目(2020A-013)资助. 
$\left.\mathrm{mAh} \cdot \mathrm{g}^{-1}\right)$ 、锌资源丰富、安全性高、成本低等特点, 得 到了研究者们的广泛关注 ${ }^{[6-11]}$. 然而, 由于多价离子在 充放电的过程中与电极材料间很强的电荷斥力和固有 的缓慢动力学, 使得真正适用于水系锌离子电池的正极 材料还比较少. 目前, $\mathrm{MnO}_{2}{ }^{[12-13]}$ 、普鲁士蓝类似物 ${ }^{[14]}$ 和 钒基化合物 ${ }^{[15-16]}$ 等正极材料已被用于锌离子的储存. 在 这些电极材料中, 具有层状、隧道结构的锰氧化物由于 具有高的理论容量 $\left(308 \mathrm{mAh} \cdot \mathrm{g}^{-1}\right)$ 、低的毒性、大电压窗 口(约 $2 \mathrm{~V}$ )和高电压输出电压(约 $1.3 \mathrm{~V})^{[17-18]}$ 等优点而备 受关注.

目前, 各种 $\mathrm{MnO}_{2}$ 相作为锌离子电池正极材料得到 研究, 例如, $\alpha-\mathrm{MnO}_{2}{ }^{[19]} 、 \gamma-\mathrm{MnO}_{2}{ }^{[20]} 、 \delta-\mathrm{MnO}_{2}{ }^{[21]}$ 等. 然而, 二氧化锰的本征导电性差 $\left(10^{-5} \sim 10^{-6} \mathrm{~S} \cdot \mathrm{cm}^{-1}\right)^{[9]}$, 且在 充放电过程中会部分溶解在电解液中, 从而影响电池的 倍率性能和循环稳定性能. 同时, $\mathrm{Zn}^{2+}$ 有着较大的半径 和电荷密度, 导致其扩散动力学相对缓慢, 并且在充放 电的过程中, $\mathrm{Zn}^{2+}$ 难以从正极中脱出, 导致不可逆的相 变, 最终造成材料结构的破坏, 也将会进一步造成电池 的容量衰减、循环稳定性和倍率性能变差. 为了解决这 些问题, 研究者通过提高二氧化锰的导电性来改善其电 化学性能. Sun 等 ${ }^{[22]}$ 利用 MOF 成功制备了含氮的导电碳 网络包覆的氧化锰纳米棒 $\left(\mathrm{MnO}_{x} @ \mathrm{~N}-\mathrm{C}\right)$, 基于该材料的 多孔结构和高效的导电网络, $\mathrm{Zn}-\mathrm{MnO}_{2}$ 电池展现出了高 的倍率性能以及循环性能. Tan 等 ${ }^{[23]}$ 在导电碳布上生长 二氧化锰, 得到高导电性和高孔隙率的正极材料, 从而 使活性材料与电解液能够充分接触, 有效地提升电子/ 离子的传输效率, 因而改善了电池的倍率性能.

综上所述, 本工作采用水热法制备出棒状结构的 $\alpha-\mathrm{MnO}_{2}$, 通过柠檬酸钠高温碳化制备多孔碳 ${ }^{[24]}$, 棒状 $\alpha-\mathrm{MnO}_{2}$ 均匀地附着在多孔碳纳米片表面得到大比表面 积的 $\alpha-\mathrm{MnO}_{2} / \mathrm{PCSs}$ 复合材料将其应用于水系锌离子电 池. 其中, 多孔碳纳米片形成的三维导电网络, 可有效 提升 $\alpha-\mathrm{MnO}_{2}$ 的电子/离子传输效率, 从而提高电池的倍 率性能. 因此, 二氧化锰的复合结构设计是进一步提高 正极材料导电性的关键.

\section{2 结果与讨论}

\section{$2.1 X$ 射线衍射 $(X R D)$ 分析, Raman 分析和 $N_{2}$ 吸脱附 分析}

图 S1 是柠檬酸钠碳化的多孔碳的 XRD 曲线, 位于 $21.84^{\circ}$ 的特征峰是多孔碳的(002)晶面. 图 1a 为 $\alpha-\mathrm{MnO}_{2}$ 和 $\alpha-\mathrm{MnO}_{2} / \mathrm{PCSs}$ 材料的 XRD 谱图, 所有的特征峰均对 应了隧道结构的 $\alpha-\mathrm{MnO}_{2}$ 的标准卡片 (JCPDS No. 44-0141) $)^{[25]}$. 尖锐的特征峰说明了 $\alpha-\mathrm{MnO}_{2}$ 具有良好的 结晶度. 经过复合后, 特征峰的强度和位置没有发生明 显的变化, 且 $\alpha-\mathrm{MnO}_{2} / \mathrm{PCSs}$ 的 XRD 曲线中没有发现碳 的特征峰, 说明样品中多孔碳纳米片大多数以无定形的
状态存在, 被较强的 $\alpha-\mathrm{MnO}_{2}$ 的衍射峰所掩盖. 图 1a 中 的插图为 $\alpha-\mathrm{MnO}_{2}$ 的晶体结构, $\alpha-\mathrm{MnO}_{2}$ 由 $\left[\mathrm{MO}_{6}\right]$ 八面体 组成, 形成了 $(2 \times 2)$ 和 $(1 \times 1)$ 隧道结构 $(2 \times 2$ 和 $1 \times 1$ 隧道 的尺寸分别为 $0.46 \mathrm{~nm}$ 和 $0.189 \mathrm{~nm})^{[19]}$. 采用拉曼光谱对 $\alpha-\mathrm{MnO}_{2} / \mathrm{PCSs}$ 进一步分析. 如图 $1 \mathrm{~b}$ 所示, 位于 555 和 $645 \mathrm{~cm}^{-1}$ 的特征峰分别对应 $\mathrm{MO}_{6}$ 基团的 $v_{3}(\mathrm{Mn}-\mathrm{O})$ 拉伸 振动和 $v_{2}(\mathrm{Mn}-\mathrm{O})$ 对称拉伸振动 ${ }^{[26]}$. 位于 1351 和 1598 $\mathrm{cm}^{-1}$ 处的两个特征峰是多孔碳的 $\mathrm{D}$ 带和 $\mathrm{G}$ 带, 分别对 应材料中的缺陷结构和石墨化结构, 并且可以用其强度 比值 $\left(I_{\mathrm{D}} / I_{\mathrm{G}}\right)$ 来说明 PCSs 的石墨化程度, $I_{\mathrm{D}} / I_{\mathrm{G}}$ 的比值为 0.75 , 表明 PCSs 石墨化程度不高, 为无定型态 ${ }^{[27]}$.

通过 $\mathrm{N}_{2}$-吸/脱附曲线对多孔碳、 $\alpha-\mathrm{MnO}_{2}$ 材料和 $\alpha-\mathrm{MnO}_{2} / \mathrm{PCSs}$ 复合材料的比表面积和孔径分布进行分 析(图 1c, $1 \mathrm{~d}$ 和图 S2), $\alpha-\mathrm{MnO}_{2}$ 和 $\alpha-\mathrm{MnO}_{2} / \mathrm{PCSs}$ 的吸/脱 附曲线表现为具有 $\mathrm{H}_{3}$ 型迟滞环的典型的 IV 型曲线, 这 种曲线类型意味着复合材料中存在介孔结构(图 $1 \mathrm{c}$ 和 $1 \mathrm{~d})$. 得益于多孔碳的高比表面积 $\left(237.92 \mathrm{~m}^{2} \cdot \mathrm{g}^{-1}\right)$ 和丰富 的孔结构(图 S2), $\alpha-\mathrm{MnO}_{2} / \mathrm{PCSs}$ 的比表面积为 $90.47 \mathrm{~m}^{2}$. $\mathrm{g}^{-1}$, 远高于 $\alpha-\mathrm{MnO}_{2}$ 的比表面积 $\left(43.43 \mathrm{~m}^{2} \cdot \mathrm{g}^{-1}\right)$. 从其孔 径分布曲线可以发现, $\alpha-\mathrm{MnO}_{2} / \mathrm{PCSs}$ 复合材料在 $2 \sim 10$ $\mathrm{nm}$ 范围内具有更丰富的介孔分布. 因而，通过制备 $\alpha-\mathrm{MnO}_{2}$ 纳米棒和多孔碳纳米片的复合材料, 极大地提 高了复合材料的比表面积. 其大的比表面积和丰富的孔 结构能够促进电解液向电极内部的快速浸润, 有效促进 锌离子的扩散.

\section{2 微观形貌分析}

图 2a $2 \mathrm{~d}$ 分别为 $\alpha-\mathrm{MnO}_{2}$ 纳米棒、多孔碳 PCSs 和 $\alpha-\mathrm{MnO}_{2} / \mathrm{PCSs}$ 复合材料的 SEM 形貌图, 图 2a 中显示 $\alpha-\mathrm{MnO}_{2}$ 具有均匀的棒状结构, 纳米棒的直径约为 50 $\mathrm{nm}$, 长度可达 $500 \mathrm{~nm}$. 图 2a 的插图是 $\alpha-\mathrm{MnO}_{2}$ 的高倍透 射电镜图(HRTEM), 可以看到其晶格间距为 $0.687 \mathrm{~nm}$, 对应了 $\alpha-\mathrm{MnO}_{2}$ 的 (110) 晶面. 因为 $\alpha-\mathrm{MnO}_{2}$ 具有 $(2 \times 2)$ 隧 道结构且该隧道的几何尺寸约为 $0.46 \mathrm{~nm}$, 所以可以容 纳 $\mathrm{Zn}^{2+}$ (尺寸约为 $0.074 \mathrm{~nm}$ ) 的嵌入/脱出 ${ }^{[28]}$. 图 $2 \mathrm{~b}$ 为 PCSs 的 SEM 图, PCSs 是由碳纳米片组成的三维多孔结 构. 图 $2 \mathrm{c}, 2 \mathrm{~d}$ 和图 $2 \mathrm{e}, 2 \mathrm{f}$ 分别是 $\alpha-\mathrm{MnO}_{2} / \mathrm{PCSs}$ 复合材料 的 SEM 图和 TEM 图, 可以看到 $\alpha-\mathrm{MnO}_{2}$ 纳米棒均匀地

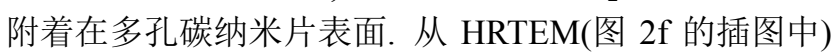
可以看出 $\alpha-\mathrm{MnO}_{2}$ 的相邻晶面的面间距为 $0.309 \mathrm{~nm}$, 对 应于 $\alpha-\mathrm{MnO}_{2}$ 的 (310)晶面, 同时可以看出 $\alpha-\mathrm{MnO}_{2}$ 与 PCSs 多孔的碳纳米片为面接触, 极大地提高了电子的 传导率, 又能够有效地避免 $\alpha-\mathrm{MnO}_{2}$ 之间的相互堆垛, 同时形成了三维导电网络, 能够有效地增强该材料在充 放电过程中电子导电性, 片层之间的间隙既能够缓冲 $\alpha-\mathrm{MnO}_{2}$ 纳米棒脱嵌 $\mathrm{Zn}^{2+}$ 过程中产生的体积变化, 又有 利于电解液的浸润. 

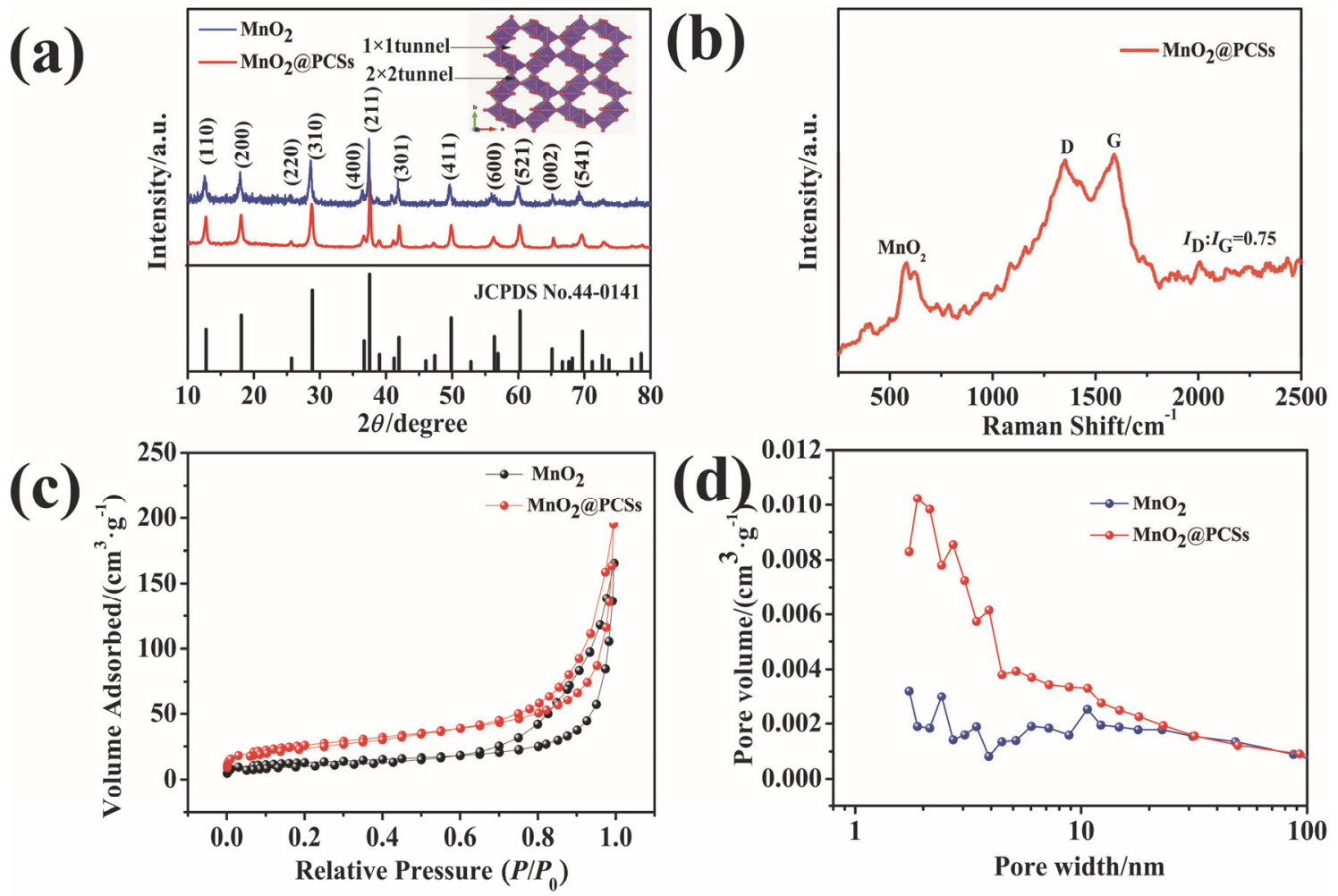

图 1 (a) $\alpha-\mathrm{MnO}_{2}$ 纳米棒和 $\alpha-\mathrm{MnO}_{2} / \mathrm{PCSs}$ 复合材料的 XRD 衍射图谱. 插图: $\alpha-\mathrm{MnO}_{2}$ 的晶体结构. (b) $\alpha-\mathrm{MnO}_{2} / \mathrm{PCSs}$ 复合材料的拉曼图谱. (c) $\alpha-\mathrm{MnO}_{2}$ 纳米棒和 $\alpha-\mathrm{MnO}_{2} / \mathrm{PCSs}$ 复合材料的 $\mathrm{N}_{2}$-吸/脱附曲线图. (d) $\alpha-\mathrm{MnO}_{2}$ 纳米棒和 $\alpha-\mathrm{MnO}_{2} / \mathrm{PCSs}$ 复合材料的孔结构分布曲线图

Figure 1 (a) XRD patterns of $\alpha-\mathrm{MnO}_{2}$ nanorods and $\alpha-\mathrm{MnO}_{2} / \mathrm{PCSs}$ composites. Inset: the crystal structure of $\alpha-\mathrm{MnO}_{2}$. (b) Raman spectrum of $\alpha-\mathrm{MnO}_{2} / \mathrm{PCSs}$ composites. (c) Nitrogen adsorption/desorption isotherms and (d) pore size distribution curve of $\alpha-\mathrm{MnO}_{2}$ nanorods and $\alpha-\mathrm{MnO}_{2} / \mathrm{PCSs}$ composites
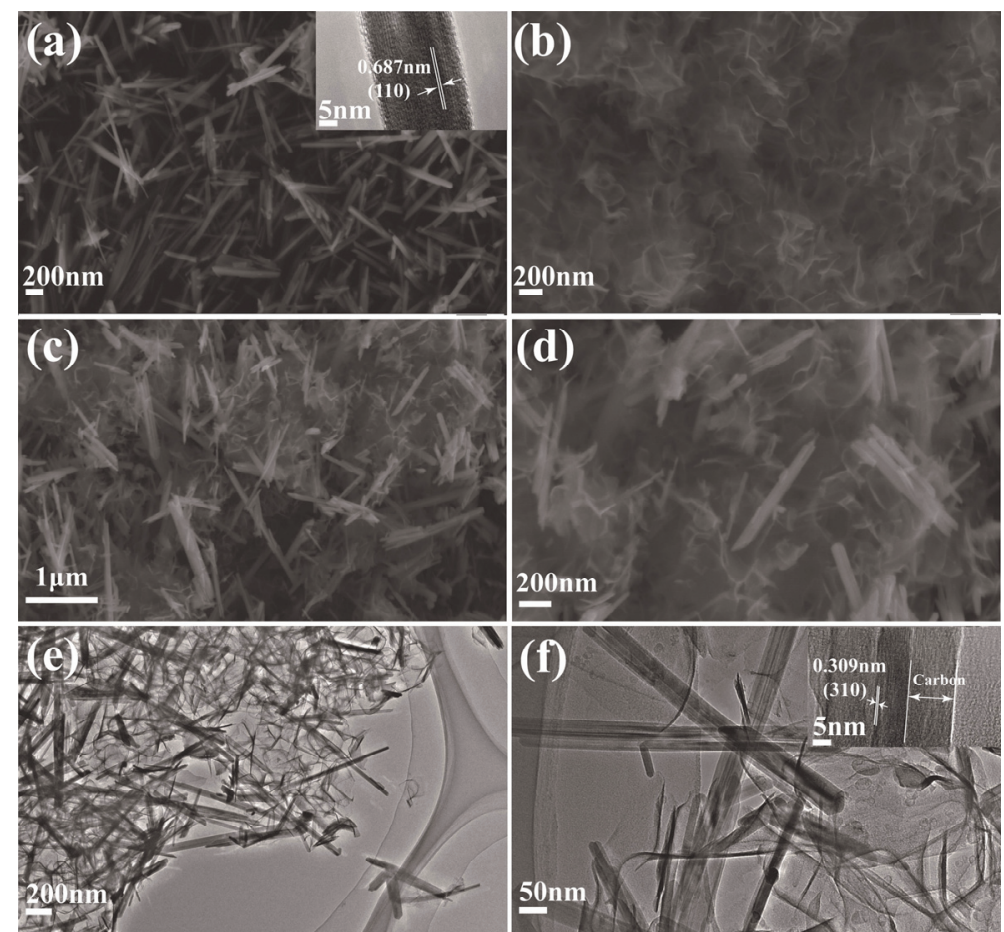

图 2 (a) $\alpha-\mathrm{MnO}_{2}$ 的 SEM. 插图: $\alpha-\mathrm{MnO}_{2}$ 的 HRTEM. (b), (c) 分别为 PCSs 和 $\alpha-\mathrm{MnO}_{2} / \mathrm{PCSs}$ 复合材料的 SEM. (d) $\alpha-\mathrm{MnO}_{2} / \mathrm{PCSs}$ 复合材料的 HRSEM. (e, f) $\alpha-\mathrm{MnO}_{2} / \mathrm{PCSs}$ 复合材料的 TEM. 插图: $\alpha-\mathrm{MnO}_{2} / \mathrm{PCSs}$ 复合材料的 HRTEM

Figure 2 (a) Scanning electron microscopy (SEM) image of $\alpha-\mathrm{MnO}_{2}$. Inset: HRTEM of $\alpha-\mathrm{MnO}_{2}$. (b), (c) SEM of PCSs and $\alpha-\mathrm{MnO}_{2} / \mathrm{PCSs}$ composites respectively. (d) $\alpha-\mathrm{MnO}_{2} / \mathrm{PCSs}$ composites. (e, f) TEM images of $\alpha-\mathrm{MnO}_{2} / \mathrm{PCSs}$ composites, inset: HRTEM of $\alpha-\mathrm{MnO}_{2} / \mathrm{PCSs}$ composites 


\subsection{XPS 分析}

为了进一步研究 $\alpha-\mathrm{MnO}_{2} / \mathrm{PCSs}$ 复合材料的元素组 成和晶体结构, 通过 $\mathrm{X}$ 射线光电子能谱 (XPS) 对 $\alpha-\mathrm{MnO}_{2} / \mathrm{PCSs}$ 复合材料进行分析. 图 $3 \mathrm{a}$ 显示 $\alpha-\mathrm{MnO}_{2} / \mathrm{PCSs}$ 复合材料表面主要由 $\mathrm{Mn} 、 \mathrm{O} 、 \mathrm{C}$ 三种元素 组成. $\mathrm{C} 1 \mathrm{~s}$ 谱图(图 3b)可以解析成位于 $284.8 \mathrm{eV} 、 285.6$ $\mathrm{eV}$ 和 $288.5 \mathrm{eV}$ 的三个峰, 分别对应于 $\mathrm{C}-\mathrm{C} 、 \mathrm{C}=\mathrm{C}$ 、 $\mathrm{C}=\mathrm{O}$ 三个价键 ${ }^{[29-30]}$. O $1 \mathrm{~s}$ 谱图(图 3c)中位于 529.8 和 531 $\mathrm{eV}$ 处的两个峰分别归因于 $\mathrm{Mn}-\mathrm{O}-\mathrm{Mn}$ 和 $\mathrm{Mn}-\mathrm{O}-\mathrm{H}^{[31]} . \mathrm{Mn}$ $2 \mathrm{p}$ 谱图(图 3d)中发现两个峰(641.88 和 $653.58 \mathrm{eV}$ ), 这与 $\mathrm{MnO}_{2}$ 的特征 $\mathrm{Mn} 2 \mathrm{p}_{3 / 2}$ 和 $\mathrm{Mn} 2 \mathrm{p}_{1 / 2}$ 结合能一致, 自旋能量 分离为 $11.7 \mathrm{eV}^{[32]}$.

\section{4 电化学性能测试}

图 $4 \mathrm{a}$ 为 $\alpha-\mathrm{MnO}_{2} / \mathrm{PCSs}$ 电极在 $1.0 \sim 1.9 \mathrm{~V}$ 电压范围 内扫描速率为 $0.2 \mathrm{mV} \cdot \mathrm{s}^{-1}$ 时的循环伏安 $(\mathrm{CV})$ 曲线. 在第 一个循环中可以观察到约 $1.1 \mathrm{~V}$ 的明显还原峰和约 1.58 $\mathrm{V}$ 的相应氧化峰. 在第二个循环中, 上述氧化还原峰值 电流降低, 可以观察到两对分离的可逆氧化还原峰(还 原峰: 1.27 和 $1.38 \mathrm{~V}$, 氧化峰: 1.54 和 $1.60 \mathrm{~V})$, 这表明电 荷存储过程分为两步. 即 $\mathrm{MnO}_{2}$ 在放电/充电过程中经历 $了 \mathrm{H}^{+}$和 $\mathrm{Zn}^{2+}$ 的嵌入/脱出过程 ${ }^{[13]}$. 与非水系电解质相比, 水系电解质中存在相当多的质子 $\left(\mathrm{H}^{+}\right), \mathrm{H}^{+}$可以参与氧化 还原反应. 因而 $\alpha-\mathrm{MnO}_{2}$ 存在 $\mathrm{H}^{+} / \mathrm{Zn}^{2+}$ 共嵌入/脱出反应 机制 ${ }^{[33]}$ :

$$
\mathrm{H}_{2} \mathrm{O} \leftrightarrow \mathrm{H}^{+}+\mathrm{OH}^{-}
$$

$$
\begin{aligned}
& \alpha-\mathrm{MnO}_{2}+\mathrm{H}^{+}+\mathrm{e}^{-} \leftrightarrow \mathrm{MnOOH} \\
& \mathrm{Zn}^{2+}+2 \mathrm{e}^{-}+2 \alpha-\mathrm{MnO}_{2} \leftrightarrow \mathrm{ZnMn}_{2} \mathrm{O}_{4}
\end{aligned}
$$

在水系 $\mathrm{Zn} / \mathrm{MnO}_{2}$ 电池中, 由于 $\mathrm{H}^{+}$和 $\mathrm{Zn}^{2+}$ 之间存在 离子半径和反应动力学、热力学的差异, $\mathrm{H}^{+}$嵌入比 $\mathrm{Zn}^{2+}$ 嵌入具有更小的过电势和电阻以及更快的离子扩散系 数, 使得 $\mathrm{H}^{+}$的嵌入比 $\mathrm{Zn}^{2+}$ 更容易, 这种 $\mathrm{H}^{+} / \mathrm{Zn}^{2+}$ 共脱/ 嵌的反应机制使 $\alpha-\mathrm{MnO}_{2}$ 具有出色的电化学性能 ${ }^{[34-35]}$. 图 $4 \mathrm{~b}$ 是 $\alpha-\mathrm{MnO}_{2}$ 和 $\alpha-\mathrm{MnO}_{2} / \mathrm{PCSs}$ 的第三次 $\mathrm{CV}$ 曲线的 对比图，两者显示了相似的氧化/还原峰，表明 PCSs 加 入不会影响 $\alpha-\mathrm{MnO}_{2}$ 电极中的氧化还原反应. 值得注意 的是, $\alpha-\mathrm{MnO}_{2} / \mathrm{PCSs}$ 电极的氧化/还原峰具有更高的峰值 电流密度, 表明其容量比 $\alpha-\mathrm{MnO}_{2}$ 电极高. 图 4c 显示了 $\alpha-\mathrm{MnO}_{2} / \mathrm{PCSs}$ 电极在 $0.1 \mathrm{~A} \cdot \mathrm{g}^{-1}$ 时的恒电流充放电曲线, 可以看到初始放电容量为 $250 \mathrm{mAh} \cdot \mathrm{g}^{-1}$, 在 $1.2 \mathrm{~V}$ 左右只 有一个平台, 这与 $\mathrm{CV}$ 曲线中首圈出现的唯一一个氧化 峰一致, 说明 $\mathrm{Zn}^{2+}$ 成功的嵌入 ${ }^{[28,36]}$. 在首次放电时 $\mathrm{Zn}^{2+}$ 嵌入隧道结构的 $\alpha-\mathrm{MnO}_{2}$ 后，一部分 $\alpha-\mathrm{MnO}_{2}$ 会不可逆地 转变为 $\mathrm{ZnMnO}_{2}{ }^{[34,37-38]}$. 在随后的循环中, 出现了两个 新的倾斜平台, 位于 $1.4 \mathrm{~V}$ 左右的斜坡是由于 $\mathrm{H}^{+}$嵌入引 起的，位于 $1.3 \mathrm{~V}$ 左右的平台是由于 $\mathrm{Zn}^{2+}$ 嵌入过程导致 的 ${ }^{[13,33]}$. 在第一个放电平台中, $\mathrm{H}^{+}$首先嵌入复合材料中, 导致电极材料周围 $\mathrm{H}^{+}$浓度降低. 随着 $\mathrm{H}^{+}$浓度降低, $\mathrm{Zn}^{2+}$ 嵌入主导电化学反应, 产生第二个放电平台 ${ }^{[13,36]}$. 经过三次循环后，可以看到充放电曲线重合，表明 $\alpha-\mathrm{MnO}_{2} / \mathrm{PCSs}$ 电极具有良好的可逆性.
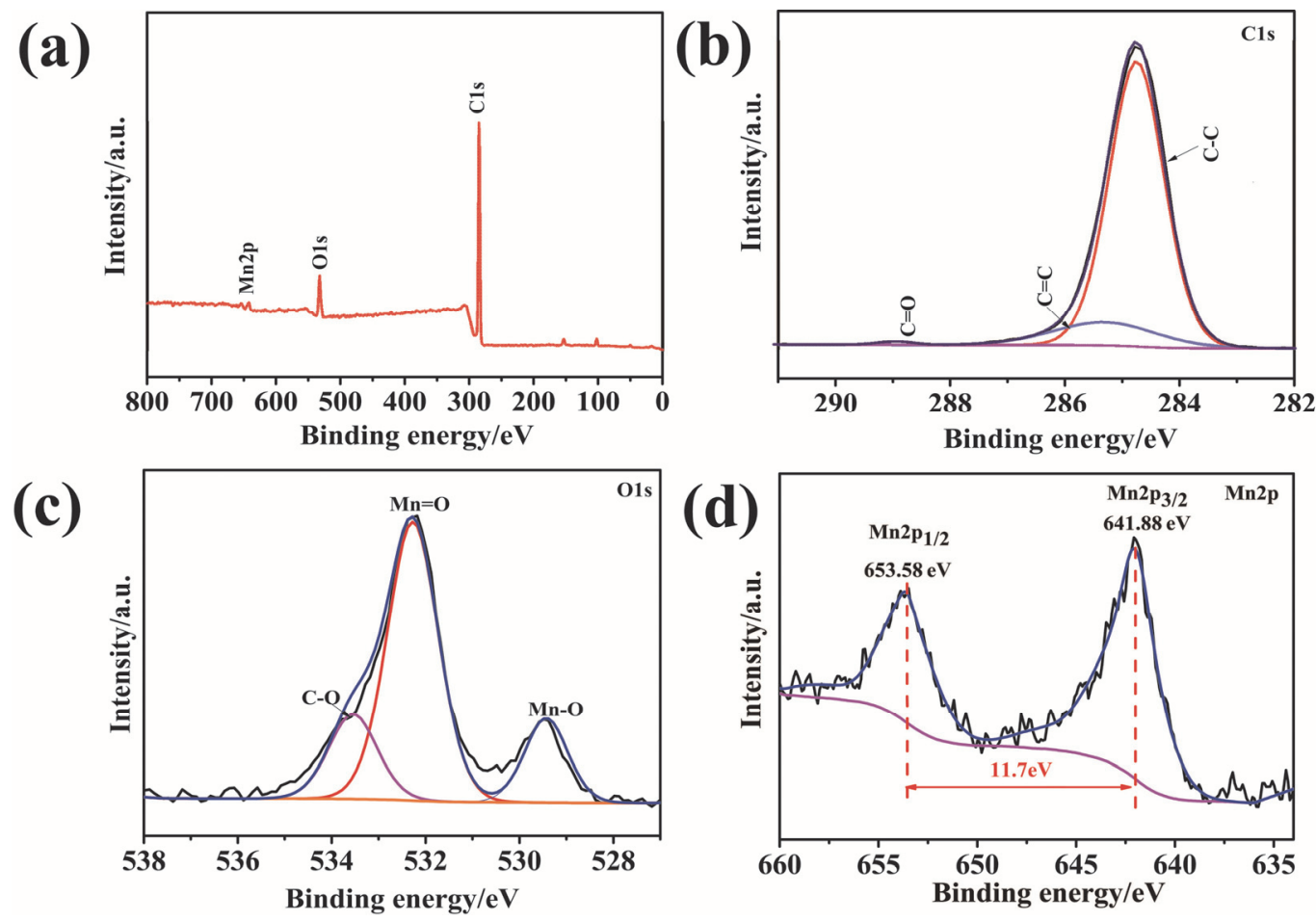

图 $3 \alpha-\mathrm{MnO}_{2} / \mathrm{PCSs}$ 复合材料的 XPS 图谱. (a) 全谱. (b) C1s 的精细图谱. (c) O1s 的精细图谱. (d) Mn2p 的精细图谱

Figure 3 XPS spectra of $\alpha-\mathrm{MnO}_{2} / \mathrm{PCSs}$ composites. (a) full spectrum. High-resolution XPS spectra of (b) C1s, (c) O1s and (d) Mn2p 

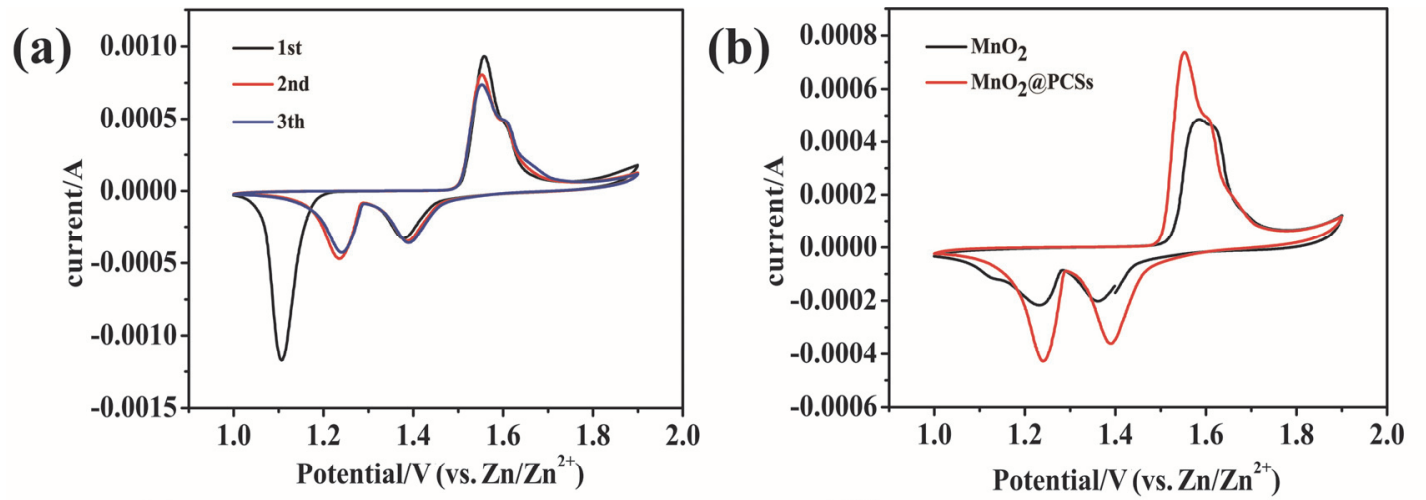

(c)

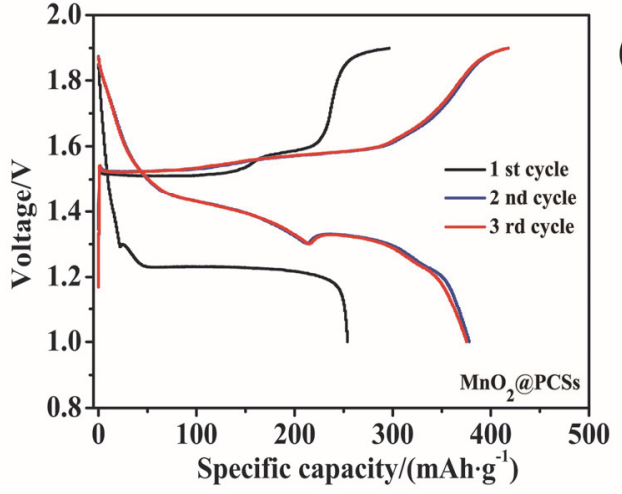

(d)

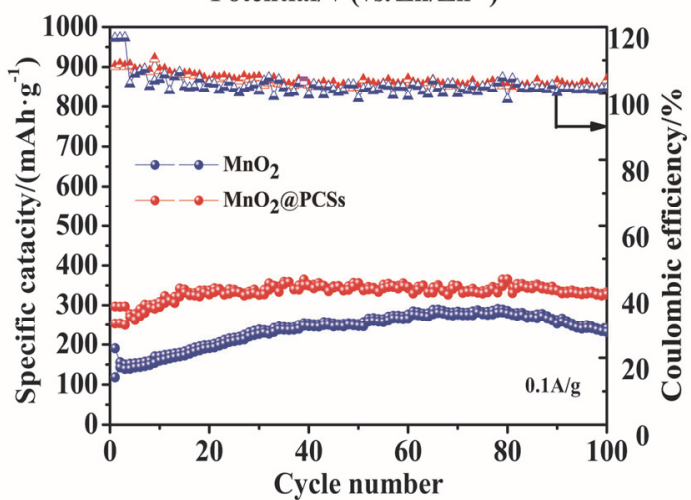

(e)

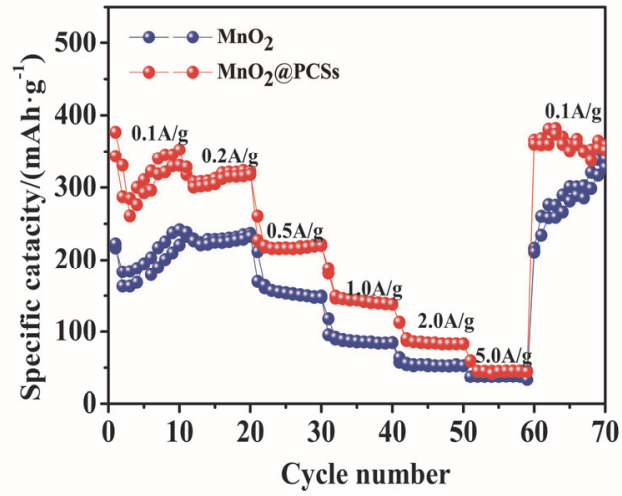

(f)

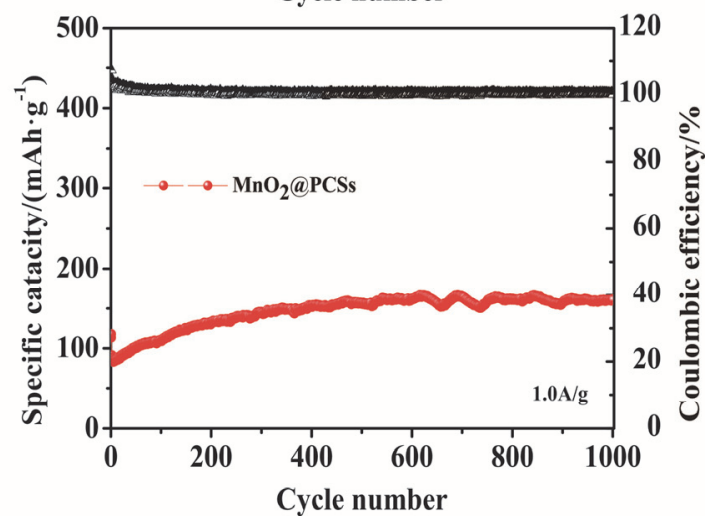

图 4 (a) $\alpha-\mathrm{MnO}_{2} / \mathrm{PCSs}$ 复合材料的前三圈的 $\mathrm{CV}$ 曲线. (b) $\alpha-\mathrm{MnO}_{2}$ 和 $\alpha-\mathrm{MnO}_{2} / \mathrm{PCSs}$ 复合材料的 $\mathrm{CV}$ 曲线. (c) $\alpha-\mathrm{MnO}_{2} / \mathrm{PCSs}$ 复合材料的前三圈充放 电曲线图. (d) $\alpha-\mathrm{MnO}_{2}$ 和 $\alpha-\mathrm{MnO}_{2} / \mathrm{PCSs}$ 复合材料的循环性能图. (e) $\alpha-\mathrm{MnO}_{2}$ 和 $\alpha-\mathrm{MnO}_{2} / \mathrm{PCSs}$ 复合材料的倍率性能图. (f) $\alpha-\mathrm{MnO}_{2} / \mathrm{PCSs}$ 复合材料的长 循环性能和库伦效率图

Figure 4 (a) Cyclic voltammetry curves of $\alpha-\mathrm{MnO}_{2} / \mathrm{PCSs}$ composites at a scan rates of $0.1 \mathrm{mV} \cdot \mathrm{s}^{-1}$. (b) Cyclic voltammetry curves of $\alpha-\mathrm{MnO}_{2}$ and $\alpha-\mathrm{MnO}_{2} / \mathrm{PCSs}$ composites. (c) The first three cycles of Charge and discharge curves of $\alpha-\mathrm{MnO}_{2} / \mathrm{PCSs}$ composites. (d) Cyclic performance of $\alpha-\mathrm{MnO}_{2}$ and $\alpha-\mathrm{MnO}_{2} / \mathrm{PCSs}$ composites. (e) Rate performance of $\alpha-\mathrm{MnO}_{2}$ and $\alpha-\mathrm{MnO}_{2} / \mathrm{PCSs}$ composites. (f) Long-term cycle performance and Coulombic efficiency of $\alpha-\mathrm{MnO}_{2} / \mathrm{PCSs}$ composites

图 $4 \mathrm{~d}$ 为 $\alpha-\mathrm{MnO}_{2}$ 和 $\alpha-\mathrm{MnO}_{2} / \mathrm{PCSs}$ 电极在电流密度 为 $0.1 \mathrm{~A} \cdot \mathrm{g}^{-1}$ 时的循环曲线. 从图中可以看出, $\alpha-\mathrm{MnO}_{2}$ 和 $\alpha-\mathrm{MnO}_{2} / \mathrm{PCSs}$ 电极在循环初期都经历了容量上涨, 不同 的是 $\alpha-\mathrm{MnO}_{2}$ 电极经过 40 次循环才趋于稳定, 80 次循环 后容量快速衰减, 100 次循环后, 容量只剩 $200 \mathrm{mAh} \cdot \mathrm{g}^{-1}$. 而 $\alpha-\mathrm{MnO}_{2} / \mathrm{PCSs}$ 电极经过 20 次循环后, 容量趋于稳定, 经过 100 次循环后, 可逆容量为 $350 \mathrm{mAh} \cdot \mathrm{g}^{-1}$, 展现了良 好的循环稳定性. 结果表明, PCSs 的存在有助于改善 $\alpha-\mathrm{MnO}_{2}$ 的循环稳定性能. $\alpha-\mathrm{MnO}_{2}$ 纳米棒均匀地附着在 多孔碳纳米片表面, 阻止了 $\alpha-\mathrm{MnO}_{2}$ 纳米棒之间相互堆 垛, 形成的三维导电网络有助于提高复合材料的电子
导电性. 图 4e 为 $\alpha-\mathrm{MnO}_{2}$ 和 $\alpha-\mathrm{MnO}_{2} / \mathrm{PCSs}$ 电极在不同电 流密度下的倍率性能, 在 $0.1 \mathrm{~A} \cdot \mathrm{g}^{-1}$ 时, $\alpha-\mathrm{MnO}_{2} / \mathrm{PCSs}$ 可 以提供放电容量约 $350 \mathrm{mAh} \cdot \mathrm{g}^{-1}$, 当电流密度增加到 5 $\mathrm{A} \cdot \mathrm{g}^{-1}$ 时, $\alpha-\mathrm{MnO}_{2} / \mathrm{PCSs}$ 的容量略高于 $\alpha-\mathrm{MnO}_{2}$. 表明 $\alpha-\mathrm{MnO}_{2}$ 与 $\mathrm{PCSs}$ 复合后, 有效地增加了复合材料在充放 电过程中容量. 因 $\alpha-\mathrm{MnO}_{2} / \mathrm{PCSs}$ 复合材料具有独特的形 貌特征, 使其在高电流密度下具有优异的长循环性能, 如图 4f 所示, 从图可以看出在 $1 \mathrm{~A} \cdot \mathrm{g}^{-1}$ 时, 经过 1000 次 循环后, $\alpha-\mathrm{MnO}_{2} / \mathrm{PCSs}$ 电极仍然可以提供约为 160 $\mathrm{mAh} \cdot \mathrm{g}^{-1}$ 的稳定容量, 达到了近 $100 \%$ 的高库仑效率. 表 明 $\alpha-\mathrm{MnO}_{2}$ 与 $\mathrm{PCSs}$ 复合后, 形成的三维多孔网络有助于 
提高电子导电性, $\alpha-\mathrm{MnO}_{2}$ 纳米棒均匀地附着在多孔碳 纳米片表面, 有效地避免 $\mathrm{MnO}_{2}$ 纳米棒的团聚, 从而提 高锌离子传输效率, 使得 $\alpha-\mathrm{MnO}_{2} / \mathrm{PCSs}$ 电极材料具有更 好的长循环稳定性能.

为了更好地理解离子传输的动力学过程, 我们在不 同的扫描速率 $\left(0.1 \sim 1.0 \mathrm{mV} \cdot \mathrm{s}^{-1}\right)$ 下进行了循环伏安测试 (图 5a). 从图可以看出, 根据动力学分析, 扩散控制与 噟电容控制共同主导电化学中的动力学过程, 随着扫描 速率的增大, 其中氧化峰和还原峰的位置向右相对移 动. 根据 $\mathrm{CV}$ 曲线中峰值电流 $(i)$ 和扫描速率 $(v)$ 之间的关

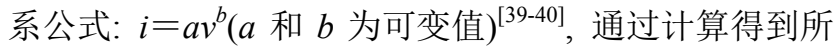
有峰值的 $b$ 值均接近 $0.5(0.64 、 0.46 、 0.41$ 和 0.52$)$, 当 $b \approx 0.5$, 这表明 $\alpha-\mathrm{MnO}_{2} / \mathrm{PCSs}$ 的容量主要受扩散过程控 制. 得益于 $\alpha-\mathrm{MnO}_{2} / \mathrm{PCSs}$ 的三维多孔结构, 可以有效缩 短 $\mathrm{Zn}^{2+}$ 的扩散距离, 从而提升了 $\mathrm{Zn}^{2+}$ 扩散速度, 有利于 扩散控制过程的电化学性能提高. 图 5c 为 $\alpha-\mathrm{MnO}_{2}$ 和 $\alpha-\mathrm{MnO}_{2} / \mathrm{PCSs}$ 电极的电化学阻抗谱图, 插图是其拟合的 等效电路图. 从图中可以看出两种材料的阻抗谱图都出 现了一个半圆(高频区)和一条斜线(低频区). 高频区对 应着电极材料中的电荷转移阻抗, 低频区对应着电极与 电解液界面离子的扩散速率 ${ }^{[41]}, \alpha-\mathrm{MnO}_{2} / \mathrm{PCSs}$ 复合材料 的斜率要远大于 $\alpha-\mathrm{MnO}_{2}$, 表明了复合材料中的离子的 扩散速率要比 $\alpha-\mathrm{MnO}_{2}$ 快. 通过等效电路模型得到相应
的动力学参数, $\alpha-\mathrm{MnO}_{2} / \mathrm{PCSs}$ 的电荷转移电阻为 228.3 $\Omega$, 远低于 $\alpha-\mathrm{MnO}_{2}$ 的电荷转移电阻(400.8 $\Omega$ ), 从而极大 地促进了 $\alpha-\mathrm{MnO}_{2}$ 的电荷转移. 为了进一步了解 $\alpha-\mathrm{MnO}_{2} / \mathrm{PCSs}$ 优异的离子扩散速率，使用恒电流间歇滴 定技术(GITT)研究了其电荷存储机理. 图 $5 \mathrm{~d}$ 示出了 GITT 曲线以及在各个反应阶段所得的扩散系数 ${ }^{[42-43]}$. 放电平台 I 的扩散系数比放电平台 II 的扩散系数高几乎 一个数量级, 表明放电平台 $\mathrm{I}$ 的 $\mathrm{H}^{+}$扩散系数比放电平台 II 的 $\mathrm{Zn}^{2+}$ 扩散系数高得多. 图 $5 \mathrm{e}$ 是计算所得的 $\alpha-\mathrm{MnO}_{2} / \mathrm{PCSs}$ 和 $\alpha-\mathrm{MnO}_{2}$ 的离子扩散系数曲线. 与 $\alpha-\mathrm{MnO}_{2}$ 相比较(扩散系数值为: $1 \times 10^{-9} \sim 1 \times 10^{-14} \mathrm{~cm}^{2}$ • $\left.\mathrm{s}^{-1}\right), \alpha-\mathrm{MnO}_{2} / \mathrm{PCSs}$ 在放电过程中显示出更大的扩散系 数值 $\left(1 \times 10^{-6} \sim 1 \times 10^{-11} \mathrm{~cm}^{2} \cdot \mathrm{s}^{-1}\right)$, 从而加快了离子扩散 速率，展现了优异的倍率性能.

\section{3 结论}

为了解决锰基材料正极材料在充放电过程中容量 低和循环稳定性差的问题, 成功构筑了一种具有三维多 孔结构的 $\alpha-\mathrm{MnO}_{2}$ 纳米棒/多孔碳纳米片正极材料. 其中, $\alpha-\mathrm{MnO}_{2}$ 纳米棒均匀地附着在多孔碳纳米片表面, 有效 地避免 $\alpha-\mathrm{MnO}_{2}$ 纳米棒的团聚, 从而提高锌离子传输效 率，增强了材料的离子扩散动力学; 三维碳网络作为高 (a)

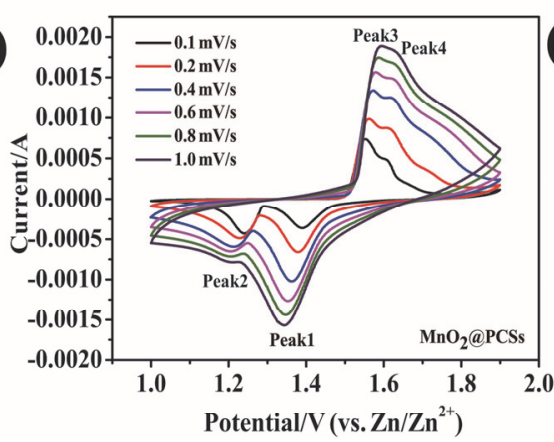

(d)

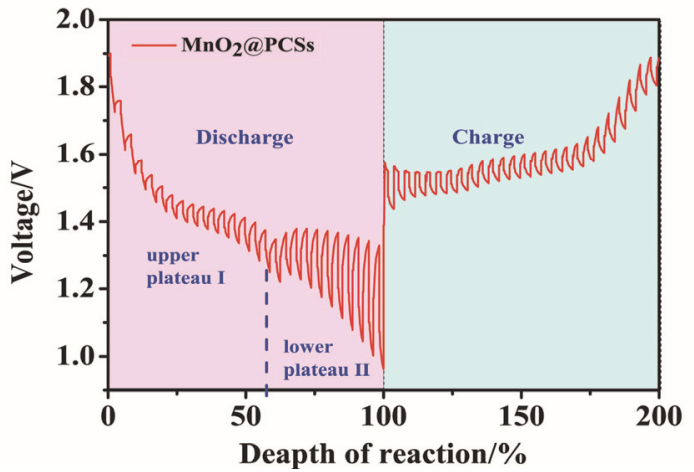

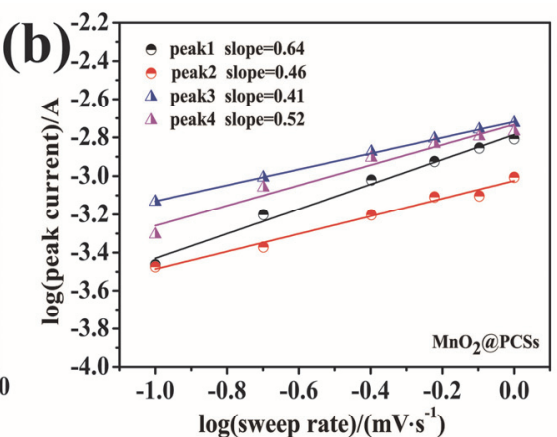

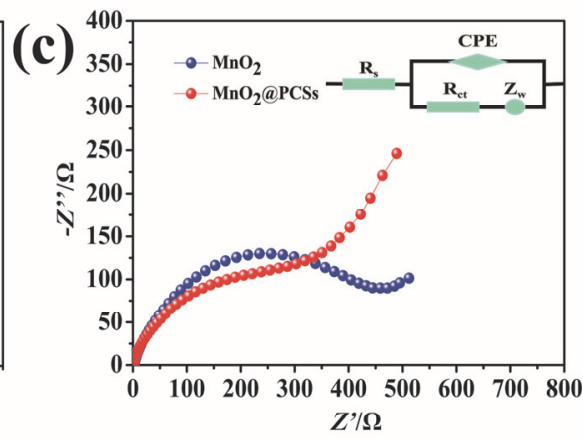

(e)

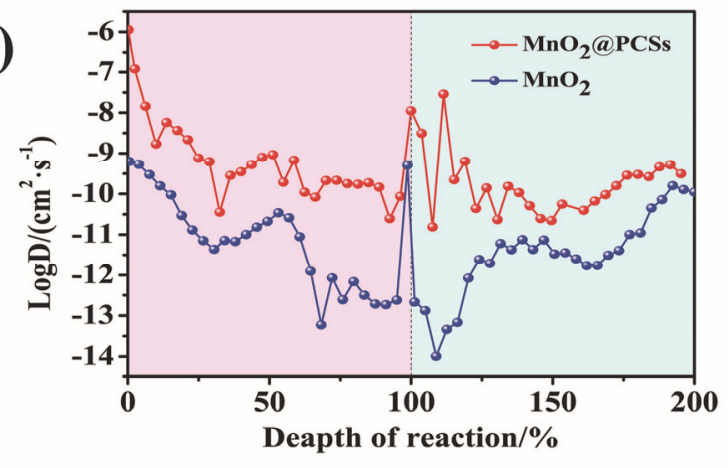

图 5 对 $\alpha-\mathrm{MnO}_{2} / \mathrm{PCSs}$ 电极的 $\mathrm{Zn}^{2+}$ 的电化学行为的动力学分析. (a) 不同电流密度 $\left(0.1 \mathrm{mV} \cdot \mathrm{s}^{-1}\right.$ 到 $\left.1.0 \mathrm{mV} \cdot \mathrm{s}^{-1}\right)$ 的 $\mathrm{CV}$ 曲线. (b) 通过峰电流和扫描速 率之间的关系来确定 $b$ 值曲线. (c) $\alpha-\mathrm{MnO}_{2}$ 和 $\alpha-\mathrm{MnO}_{2} / \mathrm{PCSs}$ 复合材料的阻抗图. (d, e) 对 $\alpha-\mathrm{MnO}_{2}$ 和 $\alpha-\mathrm{MnO}_{2} / \mathrm{PCSs}$ 复合材料的离子扩散系数进行了 GITT 测试并进行了相应的分析

Figure 5 Kinetic analysis of the electrochemical behavior of $\mathrm{Zn}^{2+}$ of $\alpha-\mathrm{MnO}_{2} / \mathrm{PCSs}$ electrode. (a) $\mathrm{CV}$ curves at various scan rates from $0.1 \mathrm{mV} \cdot \mathrm{s}^{-1}$ to 1.0 $\mathrm{mV} \cdot \mathrm{s}^{-1}$. (b) Determination of the $b$-value using the relationship between peak current and scan rate. (c) $\mathrm{Nyquist}_{\mathrm{s}}$ lot of $\alpha-\mathrm{MnO} \mathrm{O}_{2}$ and $\alpha-\mathrm{MnO}_{2} / \mathrm{PCSs}$ composites. (d, e) Calculate the ion diffusion coefficient of $\alpha-\mathrm{MnO}_{2}$ and $\alpha-\mathrm{MnO}_{2} / \mathrm{PCSs}$ composite materials GITT test was performed and corresponding analysis was performed 


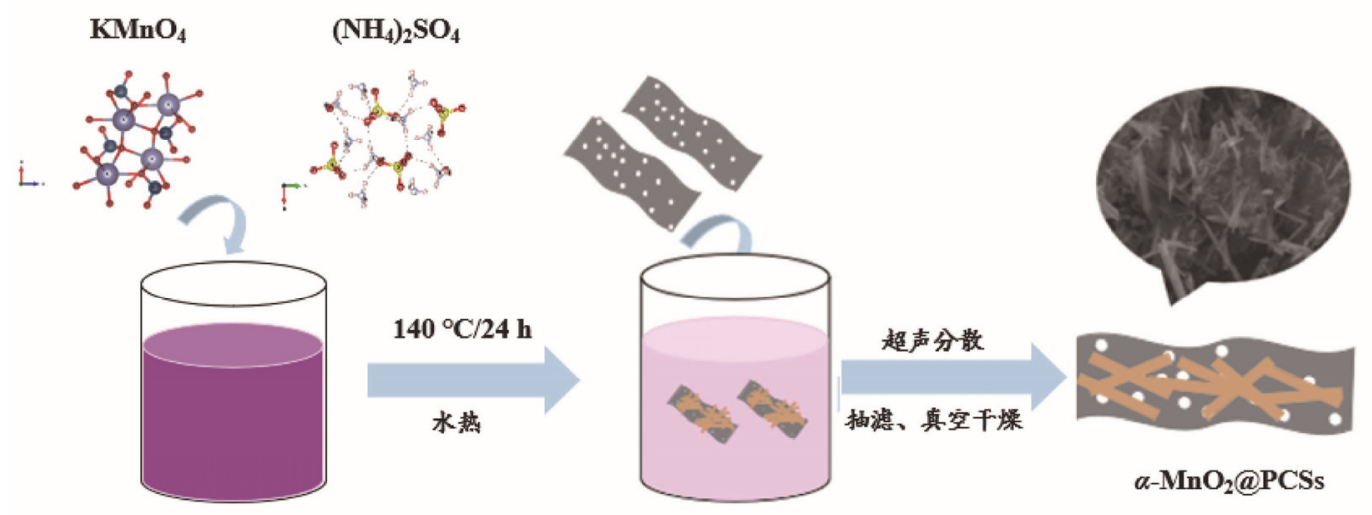

图 $6 \alpha-\mathrm{MnO}_{2} / \mathrm{PCSs}$ 复合材料的制备示意图

Figure 6 Schematic illustration for the synthesis of $\alpha-\mathrm{MnO}_{2} / \mathrm{PCSs}$ composite

导电基底, 改善 $\alpha-\mathrm{MnO}_{2}$ 导电性差的问题, 提高电极内 部电子/离子传输效率, 从而改善电池的传输动力学. 基 于其结构优势, 将其作为锌离子电池正极材料, 在电流 密度为 $0.1 \mathrm{~A} \cdot \mathrm{g}^{-1}$ 的条件下循环 100 次后, 其可逆容量为 $350 \mathrm{mAh} \cdot \mathrm{g}^{-1}$, 在 $1 \mathrm{~A} \cdot \mathrm{g}^{-1}$ 的大的电流密度下, 经过 1000 圈循环后, 容量可达 $160 \mathrm{mAh} \cdot \mathrm{g}^{-1}$, 展现了优异的循环 稳定性.

\section{4 实验部分}

\section{1 材料的制备}

\subsection{1 多孔碳( $\mathrm{PCSs})$ 的制备}

将 $5 \mathrm{~g}$ 柠檬酸钠在研钵中充分研磨 $1 \mathrm{~h}$, 然后将其置 于管式炉中, 在氩气气氛中于 $700{ }^{\circ} \mathrm{C}$ 下煅烧 $1 \mathrm{~h}$, 经洗 涤除钠盐以及干燥过程，制得多孔碳纳米片.

\subsection{2 $\alpha-\mathrm{MnO}_{2}$ 纳米棒的制备}

通过水热法来制备 $\alpha-\mathrm{MnO}_{2}$ 纳米棒. 将 $0.1264 \mathrm{~g}$ $\mathrm{KMnO}_{4}$ 和 $0.0428 \mathrm{~g}\left(\mathrm{NH}_{4}\right)_{2} \mathrm{SO}_{4}$ 溶解于 $40 \mathrm{~mL}$ 蒸馏水. 然 后将溶液倒入聚四氟乙烯内祄内, 并装入不锈钢水热反 应釜, 在 $140{ }^{\circ} \mathrm{C}$ 的温度下进行水热处理 $24 \mathrm{~h}$. 待自然降 温后, 经过过滤和蒸馏水洗涤过程. 将过滤后的粉末在 $60{ }^{\circ} \mathrm{C}$ 的真空烘箱中干燥 $24 \mathrm{~h}$, 然后在空气气氛下在 $300{ }^{\circ} \mathrm{C}$ 退火 $1 \mathrm{~h}$.

\subsection{3 $\alpha-\mathrm{MnO}_{2} / \mathrm{PCSs}$ 复合材料的制备}

$\alpha-\mathrm{MnO}_{2} / \mathrm{PCSs}$ 复合材料的制备过程如图 6 所示, 将 $40 \mathrm{mg} \alpha-\mathrm{MnO}_{2}$ 纳米棒和 $10 \mathrm{mg}$ 多孔的碳纳米片分散在 $10 \mathrm{~mL}$ 异丙醇中, 通过超声处理 $2 \mathrm{~h}$ 以形成均匀的悬浮 液. 将该悬浮液通过真空过滤得到黑色粉末, 最后, 将 黑色粉末在真空烘箱中于 $60{ }^{\circ} \mathrm{C}$ 干燥 $24 \mathrm{~h}$. 从而得到 $\alpha-\mathrm{MnO}_{2} / \mathrm{PCSs}$ 复合材料.

\section{2 分析表征}

采用扫描电子显微镜(SEM, Ultra Plus)和透射电子 显微镜(TEM, FEI Tecnia G20)对样品的微观形貌进行表
征. 通过 X 射线衍射(XRD, RigakuD/Max 2400)对样品 的晶体结构进行表征, 扫描范围: $2 \theta=10^{\circ} \sim 80^{\circ}$. 利用 拉曼光谱(RenishawIn Via Reflex Micro-Raman)分析样 品的微观结构. 元素分析是在 PHI- $570 \mathrm{X}$ 射线光电子能 谱仪(XPS)上进行的. 在 $77 \mathrm{~K}$ 的条件下以氮气吸/脱附等 温线(Micromeitics ASAP 2020)的形式表征材料的比表 面积和孔径结构.

\section{3 电化学测试}

将制备好的活性材料 $\left(\alpha-\mathrm{MnO}_{2}\right.$ 和 $\alpha-\mathrm{MnO}_{2} / \mathrm{PCSs}$ 样 品): 炭黑: 聚偏氟乙烯(PVDF)按照一定的质量比(7 : $2: 1$ )分散在 $N$-甲基-2-吡咯烷酮(NMP)中，制成均匀的 浆料, 均匀地涂敷在 $\mathrm{Ti}$ 片 $(1 \mathrm{~cm} \times 1 \mathrm{~cm})$ 表面得到电极片. 电极片在 $110{ }^{\circ} \mathrm{C}$ 真空干燥 $12 \mathrm{~h}$ 后, 以 $\alpha-\mathrm{MnO}_{2} / \mathrm{PCSs}$ 复 合材料为正极, 金属锌为负极, 以 $2 \mathrm{~mol} \cdot \mathrm{L}^{-1} \mathrm{ZnSO}_{4}+$ $0.1 \mathrm{~mol} \cdot \mathrm{L}^{-1} \mathrm{MnSO}_{4}$ 为电解液, 组装 $\mathrm{CR}-2032$ 型锌锰电 池. 电极片的活性材料的负载量为: $1.0 \sim 1.5 \mathrm{mg} \cdot \mathrm{cm}^{-2}$. 采用瑞士万通(AUTOLAB PGSTAT128N 型)的电化学工 作站对工作电极进行循环伏安测试和交流阻抗测试. 使 用蓝电测试系统(LAND-CT2001A)对不同电流密度下的 循环稳定性能、倍率性能和恒电流间歇滴定技术(GITT) 曲线进行测试.

\section{References}

[1] Yabuuchi, N.; Kubota, K.; Dahbi, M.; Komaba, S. Chem. Rev. 2014, $114,11636$.

[2] Hu, X. F.; Sun, J. C.; Li, Z. F.; Zhao, Q.; Chen, C. C.; Chen, J. Angew. Chem. Int. Ed. 2016, 128, 6592.

[3] Wang, L.; Zhao, D.-D.; Liu, X.; Yu, P.; Fu, H. G. Acta Chim. Sinica 2017, 75, 231 (in Chinese). (王蕾, 赵冬冬, 刘旭, 于鹏, 付宏刚, 化学学报, 2017, 75, 231.)

[4] Ren, T.; Zhuang, Q. C.; Hao, Y. W.; Cui, Y. L. Acta Chim. Sinica 2016, 74, 833 (in Chinese). (任粀, 庄全超, 郝玉婉, 崔永丽, 化学 学报, 2016, 74, 833.)

[5] Zhao, Q.; Yan, Z. H.; Chen, C. C.; Chen, J. Chem. Rev. 2017, 117, 10121.

[6] Lee, J.; Ju, J. B.; Cho, W. I.; Cho, B. W.; Oh, S. H. Electrochim. Acta 2013, 112, 138.

[7] Lee, B.; Lee, H. R.; Kim, H.; Chung, K. Y.; Cho, B. W.; Oh, S. H. 
Chem. Commun. 2015, 51, 9265.

[8] Häupler, B.; Rössel, C.; Schwenke, A. M.; Winsberg, J.; Schmidt, D.; Wild, A.; Schubert, U. S. NPG Asia Mater. 2016, 8, e283.

[9] Xu, D. W.; Li, B. H.; Wei, C. G.; He, Y. B.; Du, H. D.; Chu, X. D.; Qin, X. Y.; Yang, Q. H.; Kang, F. Y. Electrochim. Acta 2014, 133, 254.

[10] Li, H. F.; Xu, C. J.; Han, C. P.; Chen, Y. Y.; Wei, C. G.; Li, B. H.; Kang, F. Y. J. Electrochem. Soc. 2015, 162, A1439.

[11] Lee, B.; Yoon, K.; Seo, C. R.; Cho, B. W.; Lee, H. R.; Yoon, C. S.; Oh, S. H. ChemSusChem 2016, 9, 2948.

[12] Xu, C. J.; Du, H. D.; Li, B. H.; Kang, F. Y.; Zeng, Y. Q. Electrochem. Solid-State Lett. 2009, 12, A61.

[13] Pan, H. L.; Shao, Y. Y.; Yan, P. F.; Cheng, Y. W.; Han, K. S.; Nie, Z. M.; Wang, C. M.; Yang, J. H.; Li, X. L.; Bhattacharya, P.; Mueller, K. T.; Liu, J. Nat. Energy 2016, 1, 16039.

[14] Wang, J.; Wang, J. G.; Liu, H.; Wei, C.; Kang, F. J. Mater. Chem. 2019, 7, 13727.

[15] Alfaruqi, M. H.; Mathew, V.; Song, J. J.; Kim, S.; Islam, S.; Pham, D. T.; Jo, J.; Kim, S.; Baboo, J. P.; Xiu, Z. L.; Lee, K.S.; Sun, Y. K.; Kim, J. Chem. Mater. 2017, 29, 1684.

[16] He, P.; Yan, M. Y.; Zhang, G. B.; Sun, R. M.; Chen, L. N.; An, Q. Y.; Mai, L. Q. Adv. Energy Mater. 2017, 7, 1601920.

[17] Zeng, Y. X.; Zhang, X. Y.; Meng, Y.; Yu, M. H.; Yi, J. N.; Wu, Y. Q.; Lu, X. H.; Tong, Y. X. Adv. Mater. 2017, 29, 1700274.

[18] Qiu, W. D.; Li, Y.; You, A.; Zhang, Z. M.; Li, F. G.; Lu, X. H.; Tong, Y. X. J. Mater. Chem. 2017, 5, 28.

[19] Alfaruqi, M. H.; Gim, J.; Kim, S. J.; Song, J. J.; Jo, J.; Kim, S.; Mathew, V.; Kim, J. J. Power Sources 2015, 288, 320.

[20] Alfaruqi, M. H.; Mathew, V.; Gim, J.; Kim, S.; Song, J. J.; Baboo, J. P.; Choi, S. H.; Kim, J. Chem. Mater. 2015, 27, 3609.

[21] Alfaruqi, M. H.; Gim, J.; Kim, S.; Song, J. J.; Pham, D. T.; Jo, J.; Xiu, Z. L.; Mathew, V.; Kim, J. Electrochem. Commun. 2015, 60, 121.

[22] Fu, Y. Q.; Wei, Q. L.; Zhang, G. X.; Wang, X. M.; Zhang, J. H.; Hu, Y. F.; Wang, D. N.; Zuin, L.; Zhou, T.; Wu, Y. C.; Sun, S. H. Adv. Energy Mater. 2018, 8, 1801445.

[23] Deng, Z. H.; Huang, J. D.; Liu, J.; Ren, L.; Zhu, L. Z.; Xiao, X. Y.; Tan, M. X. Mater. Lett. 2019, 248, 207.

[24] Yan, T. T.; Xing, G. L.; Ben, T. Acta Chim. Sinica 2018, 76, 366 (in Chinese). (间婷婷, 邢国龙, 贲腾, 化学学报, 2018, 76, 366.)

[25] Tong, Z. K.; Fang, S.; Zheng, H.; Zhang, X. G. Acta Chim. Sinica 2016, 74, 185 (in Chinese). (童震坤, 方姗, 郑浩, 张校刚, 化学学
报, 2016, 74, 185.)

[26] Yang, B. B.; Wang, J.; Bin, D.; Zhu, M. S.; Yang, P.; Du, Y. K. J. Mater. Chem. 2015, B3, 7440.

[27] Huang, Y.; Liu, H.; Gong, L.; Hou, Y. L.; Li, Q. J. Power Sources 2017, 347, 29.

[28] Wang, D. H.; Li, H. F.; Liu, Z. X.; Tang, Z. J.; Liang, G. J.; Mo, F. N.; Yang, Q.; Ma, L. T.; Zhi, C. Y. Small 2018, 14, 1803978.

[29] Wang, Q. F.; Zou, R. Q.; Xia, W.; Ma, J.; Qiu, B.; Mahmood, A.; Zhao, R.; Yang, Y. Y. C.; Xia, D. G.; Xu, Q. Small 2015, 11, 2511

[30] Guo, S. P.; Li, J. C.; Ma, Z.; Chi, Y.; Xue, H. G. J. Mater. Sci. 2016, $52,2345$.

[31] Toupin, M.; Brousse, T.; Daniel, B. Chem. Mater. 2004, 16, 3184.

[32] Wang, J. J.; Wang, J. G.; Liu, H. Y.; Wei, C. G.; Kang, F. Y. J. Mater. Chem. A 2019, 7, 13727.

[33] Fang, G. Z.; Zhou, J.; Pan, A. Q.; Liang, S. Q. ACS Energy Lett. 2018, 3, 2480.

[34] Zhou, J.; Shan, L. T.; Tang, B. Y.; Liang, S. Q. Chin. Sci. Bull. 2020 65,3562 (in Chinese). (周江, 单路通, 唐博雅, 梁叔全, 科学通 报, 2020, 65, 3562.)

[35] Huang, J. T.; Zhou, J.; Liang, S. Q. Acta Phys.-Chim. Sinica 2021, 37, 2005020 (in Chinese). (黄江涛, 周江, 梁叔全, 物理化学学报, 2021, 37, 2005020.)

[36] Huang, J. H.; Wang, Z.; Hou, M.; Dong, X. L.; Liu, Y.; Wang, Y.; Xia, Y. Nat. Commun. 2018, 9, 2906.

[37] Xu, C. J.; Li, B. H.; Du, H. D.; Kang, F. Y. Angew. Chem. Int. Ed. 2012, $51,933$.

[38] Zuo, S. Y.; Xu, X. J.; Ji, S. M.; Wang, Z. S.; Liu, Z. B.; Liu, J. Chem. Eur. J. 2020, 26, 1.

[39] Wang, S. Y.; Wei, Q. L.; He, P.; Chen, Y.; Xu, X.; An, Q. Y.; Shuang, Y.; Shao, Y. Y.; Mai, L. Q.; Liu, J.; Yang, J. H. Adv. Mater. 2018, 30, 1703725 .

[40] Zhang, Y.; Deng, S. J.; Luo, M.; Pan, G. X.; Zeng, Y. X.; Lu, X. H.; Ai, C. Z.; Liu, Q.; Xiong, Q. Q.; Wang, X. L.; Xia, X. H.; Tu, J. P. Small 2019, 15, 1905452.

[41] Fen, X. M.; Huang, X. W.; Tan, Z.; Zhao, B.; Tan, S. T. Acta Chim. Sinica 2011, 69, 653 (in Chinese). (冯小明, 黄先威, 谭卓, 赵斌, 谭松庭, 化学学报, 2011, 69, 653.)

[42] Li, C. P.; Xie, X. S.; Liang, S. Q.; Zhou, J. Energy Environ. Mater. 2020, 3,146

[43] Liu, N. N.; Wu, X.; Yin, Y. Y.; Chen, A. S.; Zhao, C. Y.; Guo, Z. K.; Fan, L. S.; Zhang, N. Q. ACS Appl. Mater. Interfaces 2020, 12, 28199 .

(Cheng, B.) 